\title{
Broaching-to prevention in real-time using momentary state feedback control focusing on the saddle point in the system
}

\author{
Sreenath Maniyappan ${ }^{1} \cdot$ Naoya Umeda $^{1} \mathbb{D}$
}

Received: 3 July 2021 / Accepted: 28 December 2021 / Published online: 8 February 2022

(C) The Author(s) 2022

\begin{abstract}
For realizing real-time broaching-to prevention in regular waves, the momentary state feedback control in the vicinity of the saddle point of the dynamical system is newly proposed, based on the previous work (Maniyappan in J Mar Sci Technol, 2020, https://doi.org/10.1007/s00773-020-00743-4). This is an extension of the concept of OGY (Ott, Grebogi, and Yorke) (Ott in Phys Rev Lett 64:1196-1199, 1990) algorithm by applying momentary state feedback using the linear-quadratic regulator (LQR) only when the ship approaches a saddle point. It was numerically confirmed in regular waves that controlling the surging in a wave downslope during broaching-to enables to change the broaching to a periodic motion at lower Froude number. At higher Froude numbers, broaching-to prevention is achieved by undergoing stable surf-riding near the wave trough in lower heading angles. The robustness of the controller towards modeling uncertainties and limitation of the observed variables are also confirmed by using a controller model that ignores the nonlinear terms in the calm-water manoeuvring forces and the longitudinal position of the ship to waves.
\end{abstract}

Keywords OGY control · Controlling chaos $\cdot$ Surf-riding $\cdot$ Linear-quadratic regulator $\cdot$ Capsizing $\cdot$ Autopilot

\begin{tabular}{|c|c|c|c|}
\hline \multicolumn{2}{|c|}{ Nomenclature } & $K_{p}$ & $\begin{array}{l}\text { Derivative of roll moment with respect } \\
\text { to roll rate }\end{array}$ \\
\hline$c$ & Wave celerity & $K_{P}$ & Rudder proportional gain \\
\hline $\mathrm{C}_{\mathrm{eng}}$ & Maximum Engine torque & $K_{Q}$ & Propeller torque coefficient \\
\hline$D$ & Propeller diameter & $K_{r}$ & Derivative of roll moment with respect \\
\hline Fn & Nominal Froude number & & to yaw rate \\
\hline $\mathrm{g}$ & Acceleration due to gravity & $K_{v}$ & Derivative of roll moment with respect \\
\hline GZ & Righting arm & & to sway velocity \\
\hline$H$ & Wave height & $K_{v v v} K_{v v r} K_{v r r} K_{r r r}$ & Nonlinear derivatives of roll moment \\
\hline$I$ & $\begin{array}{l}\text { Moment of inertia of propeller and } \\
\text { shafting system }\end{array}$ & & $\begin{array}{l}\text { with respect to sway velocity and yaw } \\
\text { rate }\end{array}$ \\
\hline$I_{x x}$ & Moment of inertia in roll & $K_{w}$ & Wave induced roll moment \\
\hline$I_{z z}$ & Moment of inertia in yaw & $K_{\delta}$ & Derivative of roll moment with respect \\
\hline$J$ & Objective function & & to rudder angle \\
\hline$J_{x x}$ & Added roll moment of inertia & $K_{\phi}$ & Derivative of roll moment with respect \\
\hline$J_{z z}$ & Added yaw moment of inertia & & to roll angle \\
\hline $\boldsymbol{K}$ & Optimal feedback gain matrix & $m$ & Ship mass \\
\hline$K_{d}$ & Rudder differential gain & $m_{x}$ & Added mass in surge \\
\hline$K_{f}$ & Proportional gain of engine governor & $m_{y}$ & Added mass in sway \\
\hline & & $n$ & Propeller revolution number \\
\hline$\triangle \mathrm{N}$ & & $n_{d}$ & Desired propeller revolution number \\
\hline & g.osaka-u.ac.jp & $N_{r}$ & $\begin{array}{l}\text { Derivative of yaw moment with respect } \\
\text { to yaw rate }\end{array}$ \\
\hline 1 & $\begin{array}{l}\text { aval Architecture and Ocean Engineering, } \\
\text { of Engineering, Osaka University, 2-1 }\end{array}$ & $N_{w}$ & Wave induced yaw moment \\
\hline
\end{tabular}




\begin{tabular}{|c|c|}
\hline$N_{v}$ & $\begin{array}{l}\text { Derivative of yaw moment with respect } \\
\text { to sway velocity }\end{array}$ \\
\hline$N_{v v v} N_{v v r} N_{v r r} N_{r r r}$ & $\begin{array}{l}\text { Nonlinear derivatives of yaw moment with } \\
\text { respect to sway velocity and yaw rate }\end{array}$ \\
\hline$N_{\delta}$ & $\begin{array}{l}\text { Derivative of yaw moment with respect } \\
\text { to rudder angle }\end{array}$ \\
\hline$N_{\phi}$ & $\begin{array}{l}\text { Derivative of yaw moment with respect } \\
\text { to roll angle. }\end{array}$ \\
\hline$p$ & Roll rate \\
\hline$Q_{E}$ & Engine torque \\
\hline$Q_{P}$ & Propeller torque \\
\hline $\mathrm{R}$ & Ship resistance \\
\hline$r$ & Yaw rate \\
\hline$s$ & Control vector \\
\hline $\mathrm{t}$ & Time \\
\hline$T$ & Propeller thrust \\
\hline$T_{E}$ & Time constant of steering gear \\
\hline$u$ & Surge velocity \\
\hline$u_{f}$ & Fuel index position \\
\hline v & Sway velocity \\
\hline$x$ & State vector \\
\hline$X_{v v} X_{v r} X_{r r}$ & $\begin{array}{l}\text { Nonlinear derivatives of surge force with } \\
\text { respect to sway velocity and yaw rate }\end{array}$ \\
\hline$X_{w}$ & Wave-induced surge force \\
\hline$Y_{r}$ & $\begin{array}{l}\text { Derivative of sway force with respect to } \\
\text { yaw rate }\end{array}$ \\
\hline$Y_{v}$ & $\begin{array}{l}\text { Derivative of sway force with respect to } \\
\text { sway velocity }\end{array}$ \\
\hline$Y_{w}$ & Wave-induced sway force \\
\hline$Y_{\delta}$ & $\begin{array}{l}\text { Derivative of sway force with respect to } \\
\text { rudder angle }\end{array}$ \\
\hline$Y_{\phi}$ & $\begin{array}{l}\text { Derivative of sway force with respect to } \\
\text { roll angle }\end{array}$ \\
\hline$z_{H}$ & $\begin{array}{l}\text { Height of center of sway force due to } \\
\text { lateral motions }\end{array}$ \\
\hline$\delta$ & Rudder angle \\
\hline$\epsilon$ & $\begin{array}{l}\text { Control switching value of vicinity } \\
\text { parameter }\end{array}$ \\
\hline$\eta$ & Vicinity parameter \\
\hline$\lambda$ & Wavelength \\
\hline$\phi$ & Roll Angle \\
\hline$\chi$ & Heading angle \\
\hline$\chi_{c}$ & Desired heading angle for autopilot \\
\hline$\xi_{G}$ & $\begin{array}{l}\text { Longitudinal position of the ship center } \\
\text { of gravity in wave trough fixed coordinate } \\
\text { system }\end{array}$ \\
\hline
\end{tabular}

\section{Introduction}

The International Maritime Organization (IMO)'s operational guidance (1995) [3] suggests the shipmaster to maintain ship heading and speed outside the dangerous region in severe quartering and following seas. IMO [4] also recommends the master not to rely on automatic steering in dangerous weather, since the autopilot has not been developed to handle adverse weather scenarios. Broaching-to is one of the failure modes a ship could be vulnerable to under such dangerous conditions. The mechanism of broaching-to and its prediction has been well established by researchers over the last few decades [5, 6]. An understanding of these has led to the development of IMO guidelines for a master operating in following and quartering seas. However, these guidelines can be regarded as a passive approach involving careful observation of the sea conditions, depending to a great degree on the experience and skill of the master. This shall not always be the effective way, considering the ship's mission and particularly in an era of autonomous shipping. Hence, active methods for prevention are still an area of importance in the field of research related to broaching-to and also as a step towards improving the degree of automated control even in adverse conditions encountered in following and quartering seas.

Early attempts in the direction of broaching-to prevention had been to induce sufficient drag to prevent ship being surged by the wave. Firstly by using a sea anchor [7], but this device was observed to aggravate the situation, and later research was conducted on seabrake [7]. This approach requires structural modifications and skilled workmanship for its successful operation. Rudder effectiveness is another important factor in preventing broaching-to [8], this could be considered in the design stage by increasing the rudder area or the depth of immersion. Umeda et al. [9] introduced an autopilot system for broaching-to prevention, called the antibroaching steering system, which induces extremely large rudder angles for small deviation from the desired heading. However, the realization of this system requires a high-performance steering gear for allowing a higher maximum rudder angle limit and rudder rate. All these approaches, other than the sea anchor, require additional structural installations and hence are not easy to implement widely. A control approach that can make use of the existing steering system would be more feasible.

To date, few studies have investigated a control system approach for broaching-to prevention. The optimal control approach was attempted by Maki et al. [10] firstly by using gradient-based optimization algorithms, which were not sufficiently powerful for searching the global optimal solution in a rugged landscape. Hence a state-of-the-art optimization tool, the covariance matrix adaptation evolution strategy (CMA-ES) [11] was used by Maki et al. [12] and found effective in broaching-to prevention in regular waves. In the most recent study, the authors [1] investigated the effectiveness and mechanism of broaching-to prevention by CMA-ES using a dynamical system approach. It was understood that broaching-to prevention at lower Froude numbers is achieved 
by undergoing periodic motion and the control action near a saddle point of the dynamical system is critical in this case. And at higher Froude numbers, broaching-to prevention was achieved by undergoing surf-riding at equilibrium near a wave trough by applying a higher differential gain. However, a drawback of such an optimization strategy is the expensive computation, making it unsuitable for real-time applications. This study also showed that the unstable invariant manifold around the saddle point can take a completely different trajectory depending on the control action in this region. This behavior is similar to the OGY [2] algorithm for chaos control, which takes advantage of the large number of unstable periodic orbits existing around a chaotic attractor. Wherein, when the system is near the attractor it is pushed into the desired orbit by applying a slight perturbation to an accessible parameter. Inspired by the OGY methodology, the authors intend to provide the control action only in the vicinity of the saddle of the dynamical system so as to push the system into the desired trajectory.

This paper attempts to realize real-time broaching-to prevention by implementing the mechanism observed in optimal control using CMA-ES by adopting the concept of the OGY algorithm. A closed-loop control system is developed which momentarily evaluates the control vector by using a linearized model near the vicinity of the fixed point and applying state feedback in the rudder gains and propeller revolution using the linear quadratic regulator (LQR) [13]. A case study is carried out using this control model. Several cases where broaching-to occurred under conventional PD control are investigated by applying the new control model. The proposed controller should ideally be robust to modelling uncertainties, hence the response of the controller to modeling uncertainties is also investigated. Firstly, by using a less precise system model for the controller by omitting the nonlinear terms in calm-water manoeuvring forces. In addition, the state variable $\xi_{G} / \lambda$ representing the longitudinal position of ship center of gravity on the wave is excluded from the state variables fed to the controller. This is done to investigate the effect of such a state variable which is indeed practically difficult to be observed.

\section{Closed-loop control system schematic model}

\subsection{Momentary control concept}

The recent study [1] uncovering the mechanism of broaching-to prevention under CMA-ES revealed that at lower Froude numbers a small perturbation near the saddle point is significant in inducing a qualitative change in the trajectory of the unstable invariant manifold around the saddle point. Hence, the authors attempt to utilize this mechanism in real-time control by applying the momentary control while the system is in the vicinity of the fixed point near to a wave crest or trough. The eigenvalues shown in Fig. 1 indicate that the fixed point near to a wave crest is a highly unstable saddle and the one near to the wave trough is a weakly unstable saddle. Furthermore, in the optimal control using CMA-ES the ship experiences stable surf-riding at the fixed point near a wave trough only. Together, these results provide important insight into the nature of feedback required during the short period of the system around the fixed point. To keep the system away from the highly unstable saddle near the wave crest, the positive feedback is applied when the system is in this regime. The negative feedback trying to stabilize the system at the fixed point near a wave trough is applied when the system is in the vicinity of the fixed point near a wave trough.

\subsection{Closed-loop schematic}

Figure 2 is a schematic representation of the closed-loop system. The inputs to the system are wave parameters $(\lambda / L, H / \lambda)$, desired heading $\left(\chi_{C}\right)$ and nominal Froude number $(F n)$. Fixed points of the system are considered as an input but the same shall also be computed within the simulation model. The control vector consists of rudder gains (proportional and differential) and propeller revolutions per second (rps). The proportional-derivative (PD) autopilot and a proportional governor control the actuators, i.e. rudder, and propeller, by considering the dynamics of steering gear and shaft. The equations of coupled surge-sway-yaw-roll motion detailed in Sect. 3, evaluate the state vector. The vicinity parameter block compares the state vector with the fixed points of the system using a weighted quadratic function and the control flow is switched to either the momentary state feedback controller (lower circuit) or the normal PD control (upper circuit) based on the value of vicinity parameter. The LQR is a robust pole placement method for a linear system and allows the controller to strike a balance between

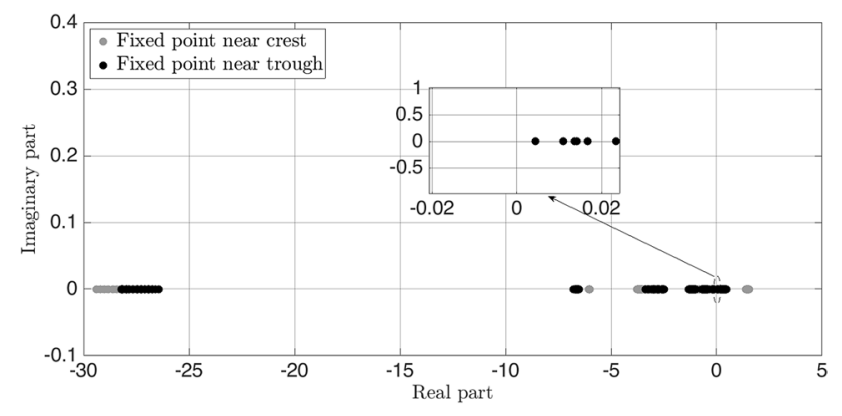

Fig. 1 Real part of eigenvalues at fixed points for a conventional PD controlled vessel modeled using a coupled surge-sway-yaw-roll model (Sect. 3) in regular waves $(\lambda / L=1.637, H / \lambda=0.1)$ for the broaching-to cases shown in Fig. 4 


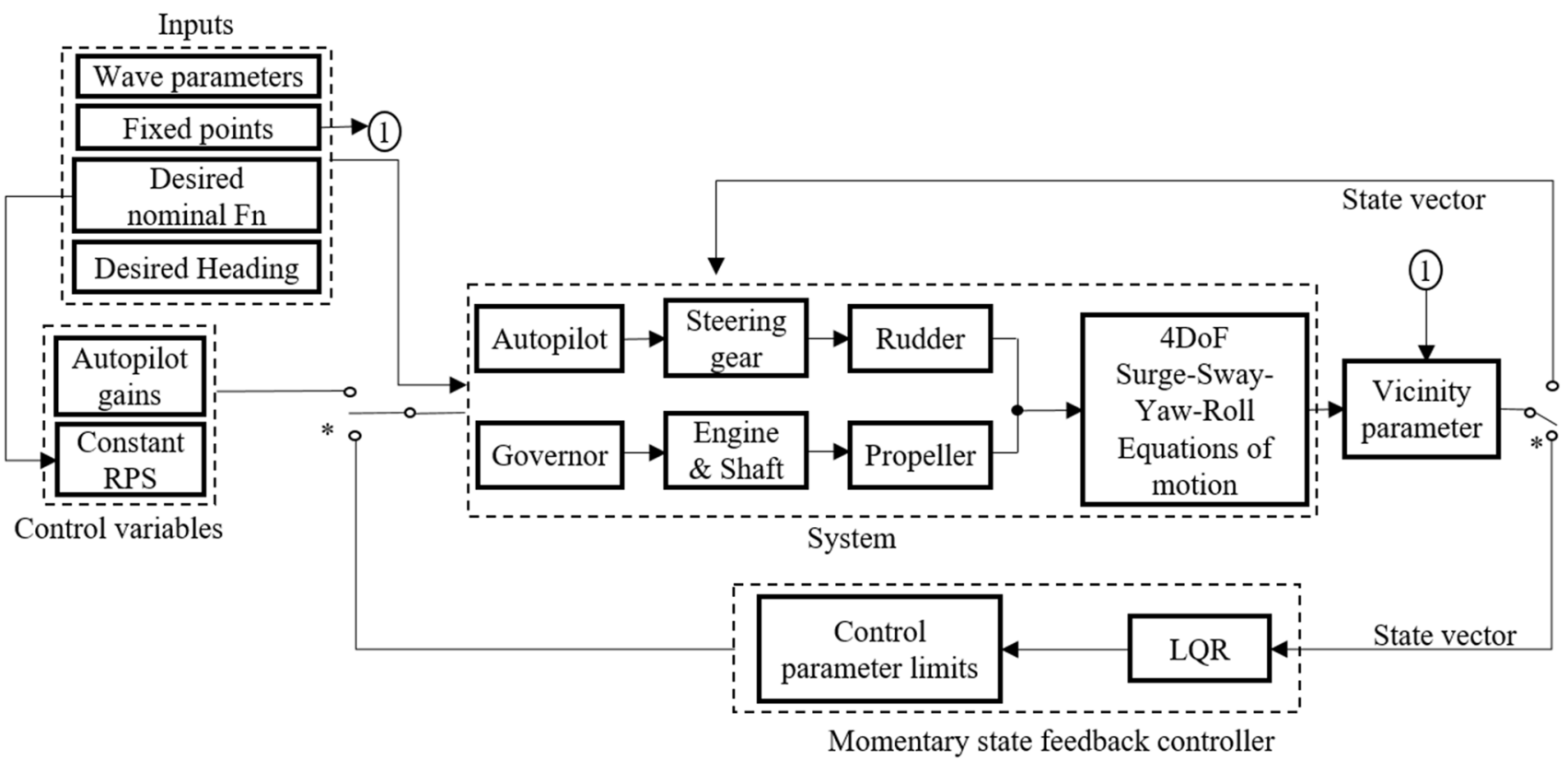

* Switch : operates based on value of vicinity parameter (turn down for $\eta<\epsilon$ )

Fig. 2 Schematic diagram of the closed-loop control system

the pole position and control effort as detailed in Sect. 3.2. The LQR controller finds the optimal feedback gain based on which the control variables are evaluated and then the limits of the control variables are applied. Conditional on the vicinity parameter value, the system either receives optimal control variables or uses the constant value for control variables. Further, the term 'proposed control model' is solely used when referring to the closed-loop control system model described in this section.

\section{Simulation model}

\subsection{System model}

The subject ship used in this study is the $34.5 \mathrm{~m}$-long Japanese purse seiner known as the ITTC Ship A-2 [14]. The maximum rudder angle to either side is 35 degrees and a maximum rudder rate of 3.5 degrees/s is considered, which is 1.5 times the SOLAS minimum requirement. The moment of inertia of the propeller shafting system used here is scaled down from that of a bulker [15], resulting in maximum engine torque to the moment of inertia ratio of propeller shafting $\left(\max \left(Q_{E}\right) / I\right)$ of $25.55 \mathrm{~s}^{-2}$. A coupled surge-swayyaw-roll model $[16,17]$ using two coordinate systems: the wave trough fixed $(\eta-\xi-\zeta)$ and an upright body-fixed $(X-Y-Z)$ are considered as shown in Fig. 3. Nonlinear terms in calm-water manoeuvring forces and shaft dynamics [15] are also included in this numerical model.

The PD controlled system is defined using the state vector, $\mathbf{x}$, and the parameter vector, $\mathbf{b}$, as follows:

$\mathbf{x}=\left\{\frac{\xi_{\mathrm{G}}}{\lambda}, u, v, \chi, r, \phi, p, \delta, n\right\}^{T}$,

$\mathbf{b}=\left\{n_{d}, \chi_{c}\right\}$

The state equations and their vector field are represented by Eqs. 3-12:

$\dot{\mathbf{x}}=\mathbf{F}(\mathbf{x} ; \mathbf{b})=\left\{\mathrm{f}_{1}(\mathbf{x} ; \mathbf{b}), \mathrm{f}_{2}(\mathbf{x} ; \mathbf{b}), \ldots, \mathrm{f}_{8}(\mathbf{x} ; \mathbf{b}), \mathrm{f}_{9}(\mathbf{x} ; \mathbf{b})\right\}^{\mathbf{T}}$,

where

$f_{1}(\mathbf{x} ; \mathbf{b})=\{u \cos \chi-\mathrm{v} \sin \chi-\mathrm{c}\} / \lambda$,

$f_{2}(\mathbf{x} ; \mathbf{b})=\left\{\mathrm{T}(\mathrm{u} ; \mathrm{n})-\mathrm{R}(\mathrm{u})+\mathrm{X}_{\mathrm{vr}}(\mathrm{u}) \mathrm{vr}+\mathrm{X}_{\mathrm{vv}}(\mathrm{u}) \mathrm{v}^{2}+\mathrm{X}_{\mathrm{rr}}(\mathrm{u}) \mathrm{r}^{2}+\left(\mathrm{m}+\mathrm{m}_{\mathrm{y}}\right) \mathrm{vr}+\mathrm{X}_{\mathrm{w}}\left(\frac{\xi_{\mathrm{G}}}{\lambda}, \chi\right)\right\} /\left(m+m_{x}\right)$ 
Fig. 3 Coordinate systems
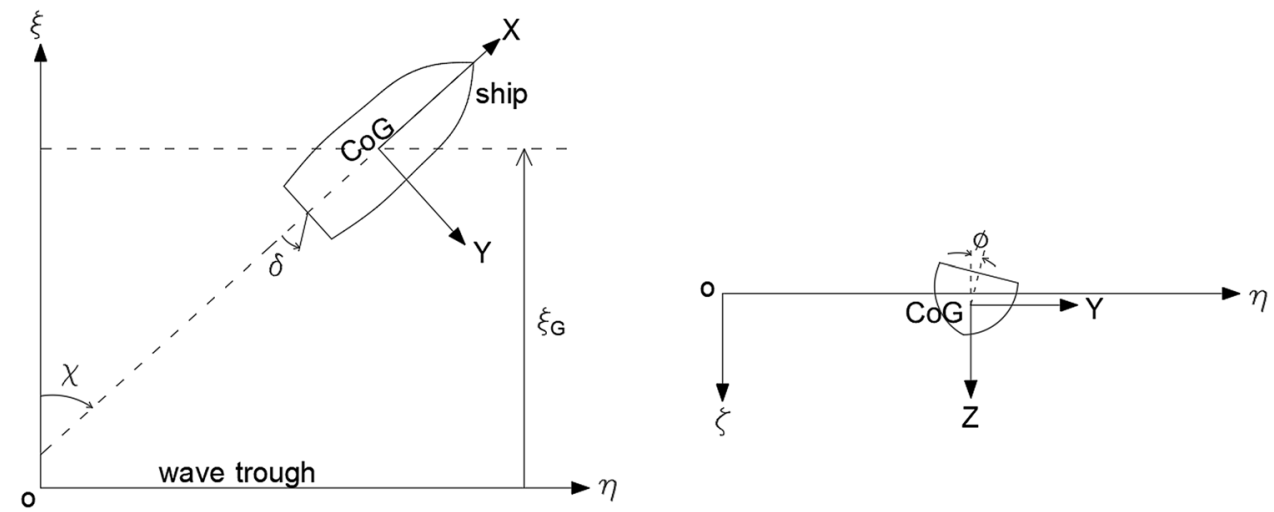

$$
\begin{aligned}
f_{3}(\mathbf{X} ; \mathbf{b})= & \left\{-\left(\mathrm{m}+\mathrm{m}_{\mathrm{x}}\right) u r+Y_{v}(u ; n) v+Y_{r}(u ; n) r+Y_{v v v}(u) v^{3}+Y_{r r r}(u) r^{3}+Y_{v v r}(u) v^{2} r+Y_{v r r}(u) v r^{2}\right. \\
& \left.+Y_{\phi}(u) \phi+Y_{\delta}(u ; n) \delta+Y_{W}\left(\frac{\xi_{G}}{\lambda}, u, \chi ; n\right)\right\} /\left(m+m_{y}\right)
\end{aligned}
$$

$$
f_{4}(\mathbf{X} ; \mathbf{b})=r,
$$

$$
\begin{aligned}
f_{5}(\mathbf{X} ; \mathbf{b})= & \left\{N_{v}(u ; n) v+N_{r}(u ; v) r+N_{v v v}(u) v^{3}+N_{r r r}(u) r^{3}\right. \\
& +N_{v v r}(u) v^{2} r+N_{v r r}(u) v r^{2}+N_{\phi}(u) \phi \\
& \left.+N_{\delta}(u ; n) \delta+N_{w}\left(\frac{\xi_{\mathrm{G}}}{\lambda}, u, \chi ; n\right)\right\} /\left(I_{z z}+J_{z z}\right),
\end{aligned}
$$

$$
\begin{aligned}
f_{7}(\mathbf{x} ; \mathbf{b})= & \left\{m_{x} z_{H} u r+K_{v}(u ; n) v+K_{r}(u ; n) r+K_{v v v}(u) v^{3}+K_{r r r}(u) r^{3}\right. \\
& +K_{v v r}(u) v^{2} r+K_{v r r}(u) v r^{2}+K_{p}(u) p+K_{\phi}(u) \phi+K_{\delta}(u) \delta \\
& \left.+K_{w}\left(\frac{\xi_{G}}{\lambda}, u, \chi: n\right)-m g G Z(\phi)\right\} /\left(I_{x x}+J_{x x}\right),
\end{aligned}
$$$$
f_{8}(\mathbf{x} ; \mathbf{b})=\left\{-\delta-\mathrm{K}_{P}\left(\chi-\chi_{c}\right)-K_{d} r\right\} / T_{E},
$$

$f_{9}(\mathbf{x} ; \mathbf{b})=\left\{Q_{E}-Q_{p}\right\} / 2 \pi I$,

$u_{f}=K_{f}\left(n_{d}-n\right)$,

$Q_{E}=u_{f} C_{e n g}: u_{f}=[0,1]$,

$Q_{p}=K_{Q}(J) \rho n^{2} D^{5}$

The symbols used in Eqs. 1-15 are explained in the nomenclature. Equation 12 considers the shaft dynamics. A simplified engine model assuming a linear relationship between fuel supply and engine torque is used to obtain the engine torque Eq. 14 . Here the parameter $u_{f}$ indicates the fuel rack position ranging from zero to one evaluated using a proportional governor Eq. 13 .

\subsection{Momentary state feedback controller}

A nonlinear system shall behave similarly to its linearized approximation for a small range of motions. This fundamental idea supported by Lyapunov's linearization methods enables the use of linear control techniques on nonlinear systems. Further, Fig. 1 shows that the real part of eigenvalues $(\lambda)$ at the fixed points of this system is always away from zero i.e. $\operatorname{Re}(\lambda) \neq 0$, this follows the Hartman-Grobman theorem [18] for topological equivalence of a linearized model to the nonlinear system in that small region. The state feedback controller used here is based on this conceptual framework. In the vicinity of the fixed points, a linearized model based on the 4 degrees-of-freedom (DoF) model is used for the system. The control feedback using LQR is applied to this linearized model near the fixed point $\boldsymbol{x}^{*}$ with control vector $\boldsymbol{s}^{*}$, mathematically described by Eqs. 16-18:

$\dot{\mathbf{x}}_{t}-\dot{\mathbf{x}}^{*}=A\left(\mathbf{x}_{t}-\mathbf{x}^{*}\right)+B\left(s-s^{*}\right), \forall \eta \leq \epsilon$,

$\boldsymbol{s}=\left[K_{P} K_{d} n_{d}\right], K_{P} \in[0,3], K_{d} \in[0,900 \mathrm{~s}], n_{d} \in\left[0, n_{\max }\right]$,

$\delta s=s-s^{*}= \pm K \delta x$,

$\eta=\delta \mathbf{x} W \delta \mathbf{x}$

At the fixed point $\mathbf{x}^{*}, \dot{\mathbf{x}}^{*}$ evaluates to zero in Eq. 16 . The term $\delta \boldsymbol{x}\left(\delta \boldsymbol{x}=\mathbf{x}_{t}-\mathbf{x}^{*}\right)$ denote the deviation of state vector from fixed point and $\delta s$ denote the additional control effort. The matrices $A$ and $B$ are the Jacobians in Eqs. 22 and 23 concerning the state vector and the control vector, 
respectively. The control vector, $\boldsymbol{s}$, with the control variables, i.e. $K_{P}$, rudder proportional gain, $K_{d}$, rudder differential gain, and $n_{d}$, the desired rps. Here, the rudder is controlled by tuning the rudder gains. The importance of tuning rudder gains in broaching-to prevention is brought out by previous researches $[1,9]$. This also helps to maintain the course-keeping objective when switching to the proposed control model. The limits of the rudder gains are based on the manufacturers' data used for commercial autopilots as given by Ohtsu and Hasegawa [19]. These limits are applied to the state feedback Eq. 18 obtained using the LQR. The symbol $n_{\max }$ denotes the rps corresponding to surge velocity at surf-riding equilibrium. The symbol $\eta$ is the vicinity parameter, and $\epsilon$ is the value of $\eta$ below which the momentary state feedback control is applied. The symbol $\boldsymbol{K}$ is the LQR feedback gain and $W$ is the weighted diagonal matrix for defining the vicinity parameter with higher weight given in surge direction. Typical value of the diagonal elements

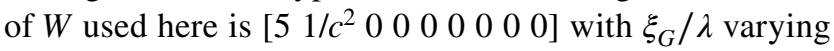
from 0 to 1 . These values and the $\epsilon$ value are chosen hand in hand by trial and error to ensure that there is clear change in the vicinity parameter $(\eta)$ when the system moves from one fixed point to the other.

The linear quadratic regulator (LQR) can be forced to satisfy any desired set of poles with appropriate linear feedback. The LQR is an optimal design technique that guarantees a robust system [20] and enables the designer to tune the control effort and position of poles. The objective function $J$ with diagonal weight matrix $Q$ and $R$ is optimized by solving the matrix algebraic Riccati equation to give feedback gain matrix $\boldsymbol{K}$ [13]. State feedback is given by $\delta \boldsymbol{s}=-\boldsymbol{K} \delta \boldsymbol{x}$ which minimize the quadratic cost function shown in Eq. 20. The same cost function is used when the ship is near to wave crest or wave trough fixed point. This means, the feedback gain matrix $\boldsymbol{K}$ attempts to stabilize the system at the fixed point by applying negative feedback. However as discussed in Sect. 2.1 the fixed point near wave crest is highly unstable and hence positive feedback is applied when the system is near wave crest fixed point as represented by Eq. 18 to keep the system away from this fixed point.

$J=\int_{0}^{\infty}\left(\delta \mathbf{x}^{T} Q \delta \mathbf{x}+\delta \boldsymbol{s}^{T} R \delta \boldsymbol{s}\right) d t$,

$Q$ positive semi definite $; R$ positive definite.

subject to system dynamics.

$\delta \dot{\mathbf{x}}=A \delta \mathbf{x}+B \delta \boldsymbol{s}$,

$A=\frac{\partial}{\partial \mathbf{x}_{\mathbf{j}}} f_{i}(\mathbf{x} ; \mathbf{b}), 1 \leq i, j \leq 9$,

$B=\frac{\partial}{\partial s_{\mathrm{j}}} f_{i}(\mathbf{x} ; \mathbf{b}), 1 \leq i \leq 9$ and $1 \leq j \leq 3$,

The choice of weight matrix $\mathrm{Q}$ and $\mathrm{R}$ in Eq. 20 should be such that to ensure maximum control effort is applied, focusing mainly on the surge and yaw dimensions of the system. As an example, in this study the

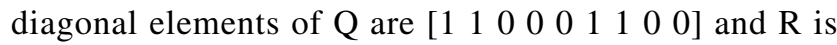
$\left[1 / K_{p \max }^{2} 0.01 / K_{d \max }^{2} 0.1 / n_{\text {const }}^{2}\right]$ where ' $\max$ ' refers to the maximum value of the respective gains and ' $n_{\text {const }}$ ' refers to the rps corresponding to the nominal Froude number. It shall be noted here that it is only important to use values of similar proportion.

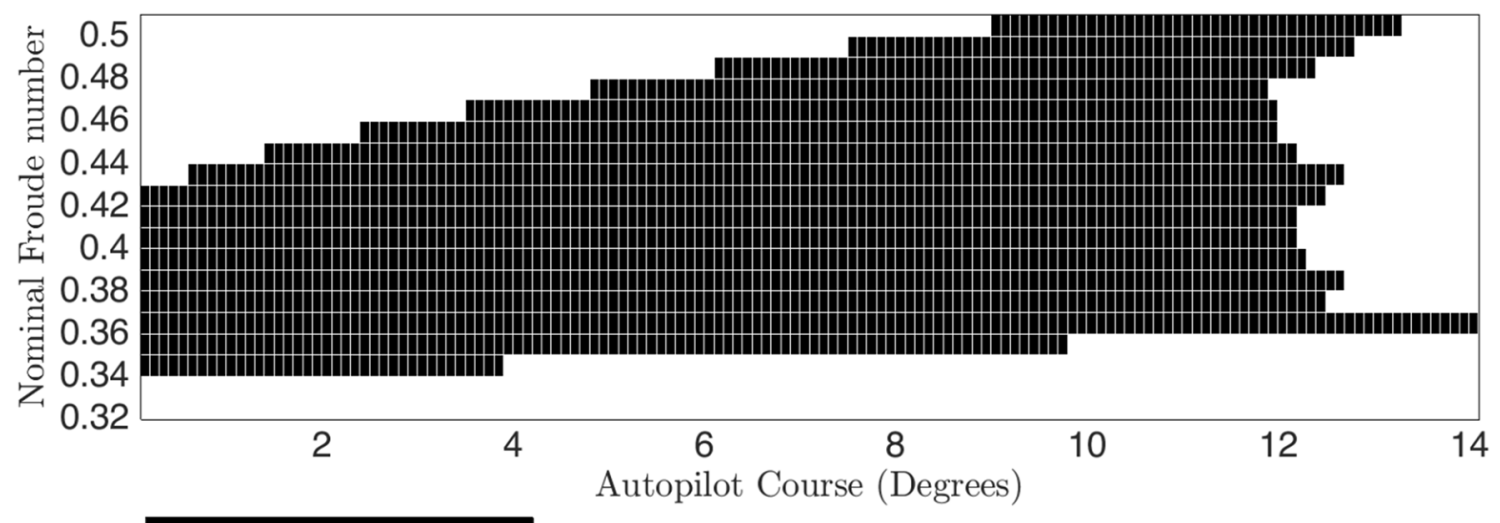

Broaching-to and capsize

Fig. 4 Capsize due to broaching-to region under the conventional PD control with $\lambda / \boldsymbol{L}=1.637$ and $\boldsymbol{H} / \boldsymbol{\lambda}=0.1$ 


\section{Numerical results and discussion}

Numerical simulation using the proposed control model is carried out for capsizing due to broaching-to cases shown in Fig. 4 in regular waves, in which $\lambda / L_{B P}=1.637$ and $H / \lambda=0.1$, occurring under conventional PD control. Here the operational parameters to be investigated are the nominal Froude number, which is the Froude number in calm-water realized with the target propeller revolution, and the desired heading angle of the PD autopilot from the wave direction. The control parameters for the conventional PD control are $K_{P}=3.0$ and $K_{d}=18.081$ obtained by optimizing the course-keeping objective function [1] for a particular periodic motion case $\left(F n=0.3, \chi_{c}=20\right)$ using CMA-ES. The choice of rudder gains will affect the comparison between conventional PD and the proposed control model. However, conventional PD control mostly uses a constant value for rudder gains, and hence use of such optimized value here could result in a reasonable comparison. The proportional governor for the engine fuel index uses a constant gain value of $K_{f}=2$, chosen by trial and error to keep up to the desired rps and more importantly to provide more periodic motion cases with the proposed control model. Firstly, the capsize due to broaching-to cases under the PD control are identified following the criteria proposed by Umeda and Renilson [5, $9,16]$, by carrying out numerical simulation. Initial conditions for this were chosen based on the sudden change concept (SCC) [21] to take into account the dependence on the initial condition and avoid impractical initial states.
Figure 4 shows the capsize due to broaching-to cases identified at steps of 0.01 for the nominal Froude number and 0.1 degrees desired heading intervals from the wave direction. Outside the capsize due to the broaching-to region indicated in Fig. 4, the ship undergoes other modes of motion such as a periodic motion for lower Froude numbers, surf-riding in higher Froude number with the low heading angle, and capsize without broaching-to in higher heading angles which are not relevant in this study. The broaching-to cases in the Froude number range between 0.34 to 0.5 , and autopilot course of up to 14 degrees are considered for further simulations to investigate using the proposed control model.

\subsection{Comparison between the conventional PD control and the proposed control model}

Capsize due to broaching-to cases (Fig. 4) are analyzed by applying the proposed control model and the results are compared with the conventional PD control. In this section, the authors discuss two representative cases exhibiting unique mechanisms of broaching-to prevention, case I at a lower Froude number and case II at a higher Froude number.

\subsubsection{Case I: $F n=0.35, \chi_{c}=6^{\circ}$}

Figure 5a-f shows that periodic motion occurs under the proposed control model, and the system goes into capsize due to broaching-to under the PD control (time 0 to $40 \mathrm{~s}$ ). During the time interval of 30 to $40 \mathrm{~s}$, under the PD control
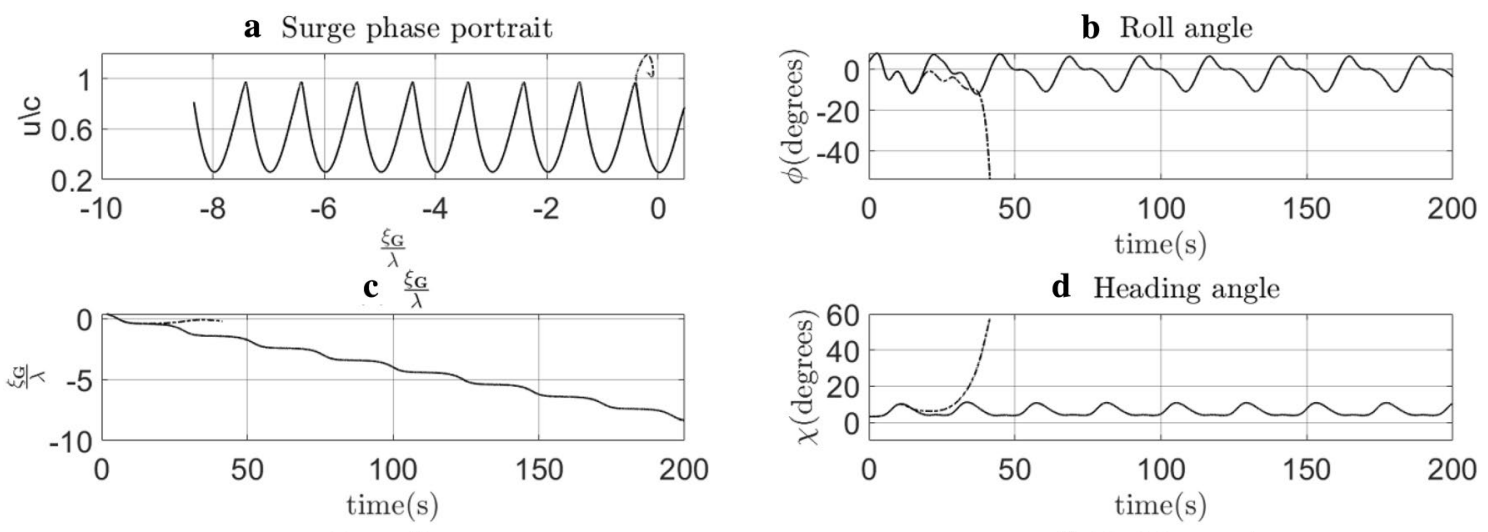

e Propeller rps
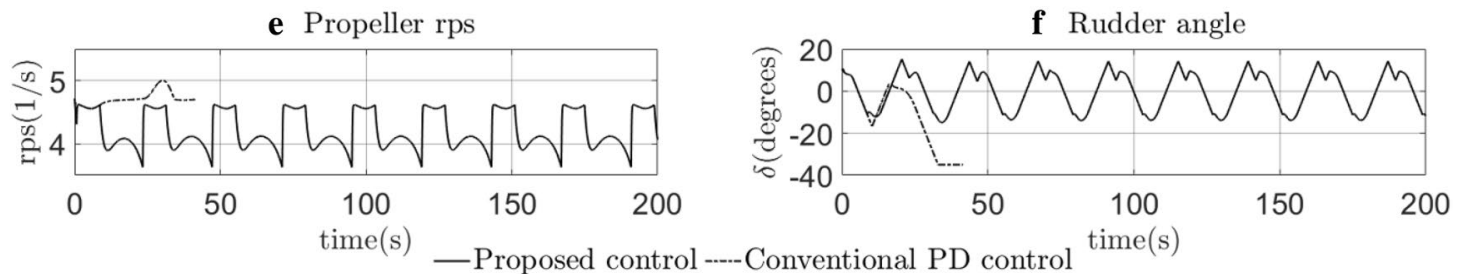

Fig. 5 Case I: comparison between the conventional PD control and the proposed control model for the case I 

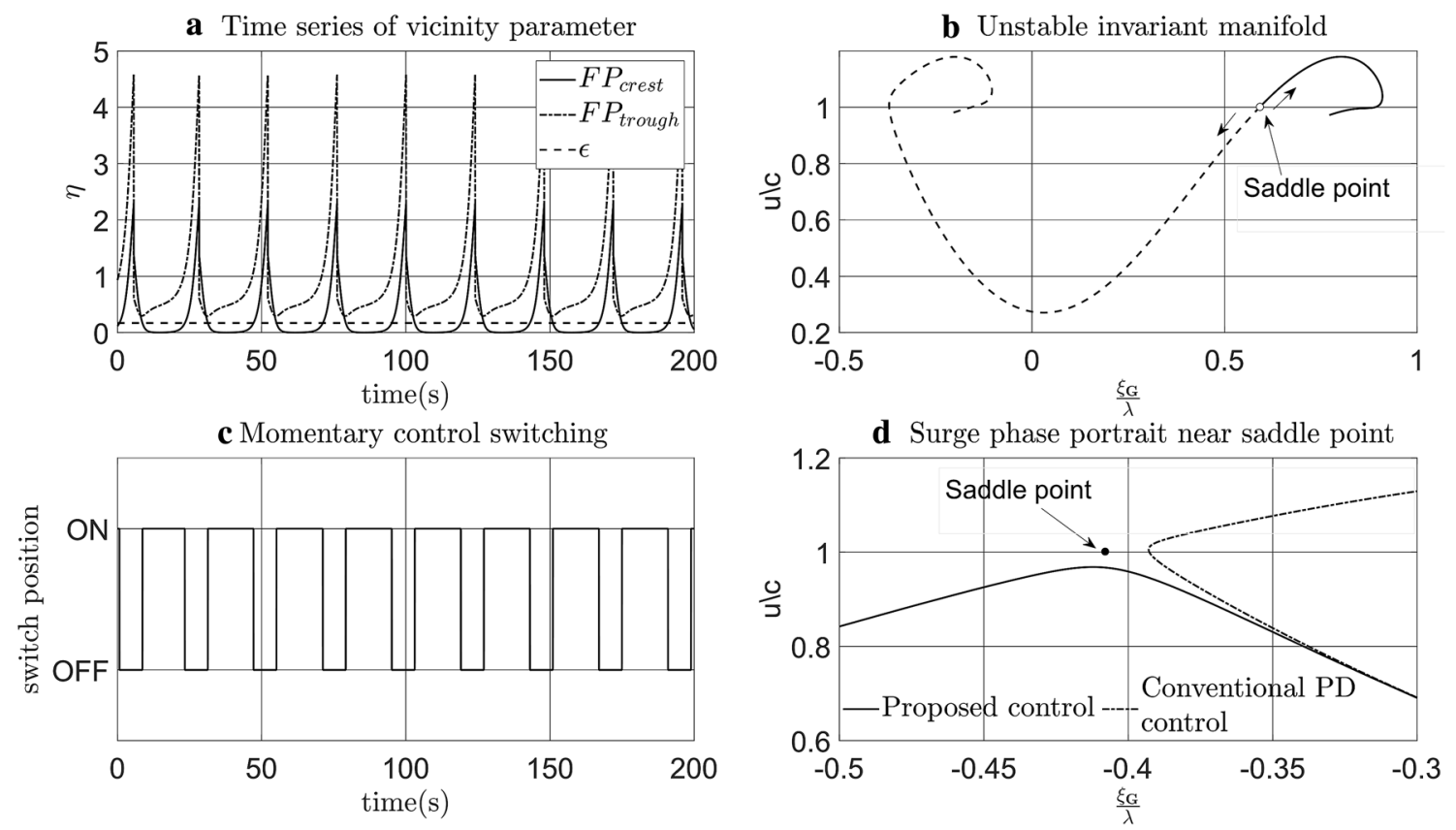

Fig. 6 Case I: Vicinity parameter, control switch time histories and surge phase planes

the surge velocity increases beyond the wave celerity around the saddle near a wave crest $\left(\xi_{G} / \lambda=-0.5\right)$ as shown by Fig. $5 \mathrm{a}$ and c, then leads to large yawing (Fig. $5 \mathrm{~d}$ ) to starboard on the wave downslope and roll angle (Fig. 5b) increases in the opposite direction due to the centrifugal force. The rps spikes (Fig. 5e) near the wave trough due to the high inflow velocity which increases the advance velocity and reduces propeller torque. The rudder turns to a hard port (Fig. 5f) at around $30 \mathrm{~s}$ to counter the significant yawing towards starboard and the ship finally capsizes as the roll angle reaches the angle of vanishing stability. In the proposed control model, as the system reaches near the wave crest saddle, the additional control action is switched $\mathrm{ON}$ as shown by Fig. 6a and c. Figure 6a indicates the proximity of the system to either of the fixed points in which the control action is taken accordingly. $F P_{\text {crest }}$ shows the $\eta$ value concerning to fixed point near a wave crest and $F P_{\text {trough }}$ shows the same in relation to a wave trough fixed point. The surging is controlled by the reduction in rps (Fig. 5e) and larger rudder angle (Fig. 5f) induced by the LQR control, whenever the $\eta$ value for a wave crest is lower than the $\varepsilon$ value. Figure $6 \mathrm{~b}$ shows the unstable invariant manifold of the saddle point near the wave crest. Figure $6 \mathrm{~d}$ is the close-in view of the phenomenon happening near the saddle indicating that a slight control action near the saddle makes the system follow a completely different trajectory.

\subsubsection{Case II: $F n=0.44, \chi_{c}=2^{\circ}$}

Figure 7a-f shows that capsize due to broaching happens under the PD control and surf-riding happens under the proposed control model. In the PD control the surging happens in the wave downslope as shown by Fig. 7a and c combined with large yaw (Fig. 7d) causes the ship to a large roll (Fig. 7b) during the time interval of around 50 to $80 \mathrm{~s}$. The rudder angle (Fig. 7f) reaches its saturation value in an attempt to control the yaw and finally capsize occurs as the roll angle reaches the angle of vanishing stability. In the case of the proposed control model, as the system falls into the fixed point near the wave trough shown by Fig. 8a, the control switches from the PD to the LQR applying the large rps (Fig. 7e) resulting in higher propeller and rudder force to stabilize the system at the fixed point near the wave trough. The surge phase portrait (Fig. 7a) shows that the system falls into a surf-riding motion near the wave trough. However, the feasibility of providing the higher rps depends on the engine specification.

Figure 9 shows the summary of the wider case study carried out using the proposed control model. The broaching-to prevention by undergoing the periodic motion is achieved for $F n=0.34$ to 0.36 and by the surf-riding is observed in higher Froude numbers. It shall also be noted here that the definition of high and low Froude number will vary depending on the wavelength. Because a higher wave celerity will increase the surf-riding threshold. However, at large heading angles the proposed control model fails to prevent broaching-to. It shall be noted here that the 

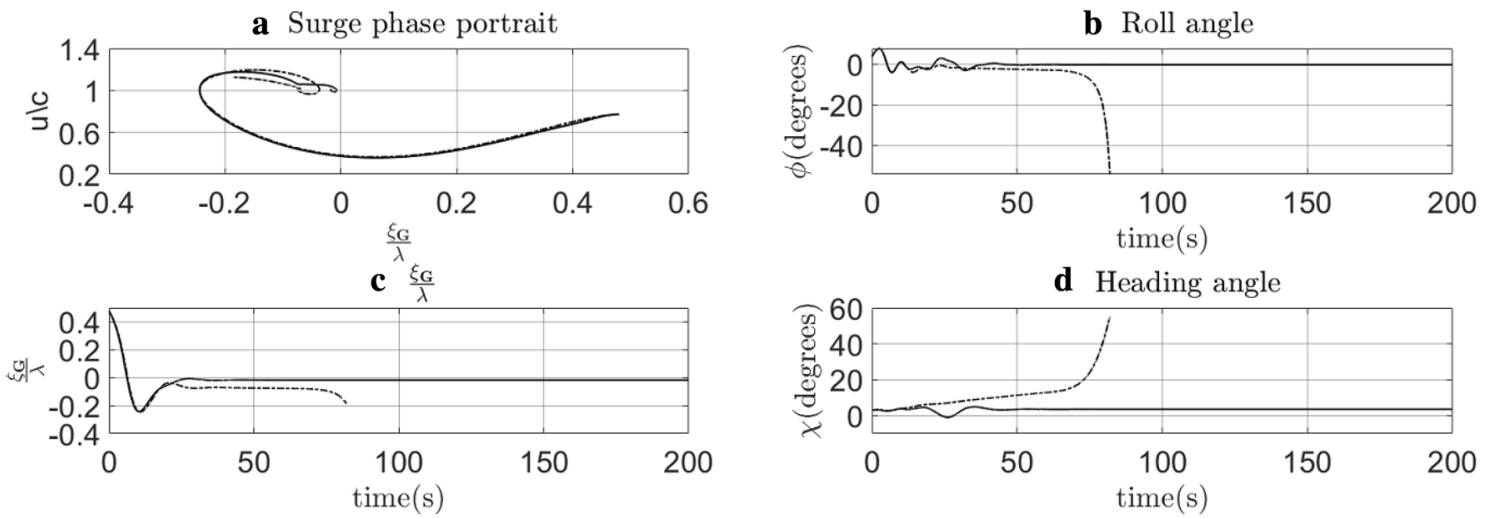

d Heading angle

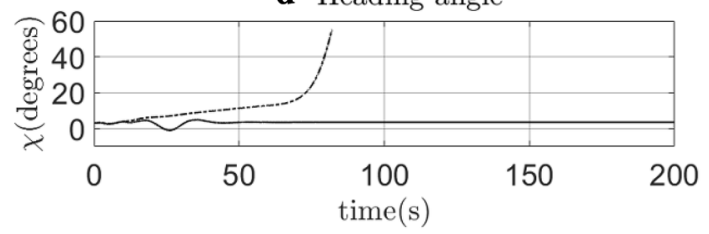

e Propeller rps

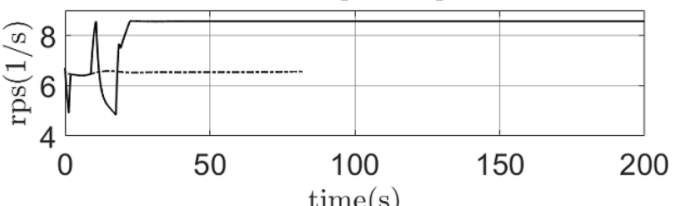

f Rudder angle

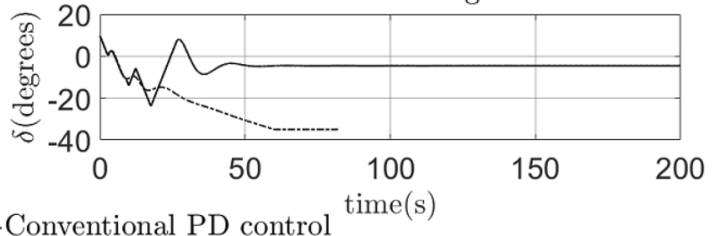

Fig. 7 Case II: comparison between conventional PD control and proposed control model
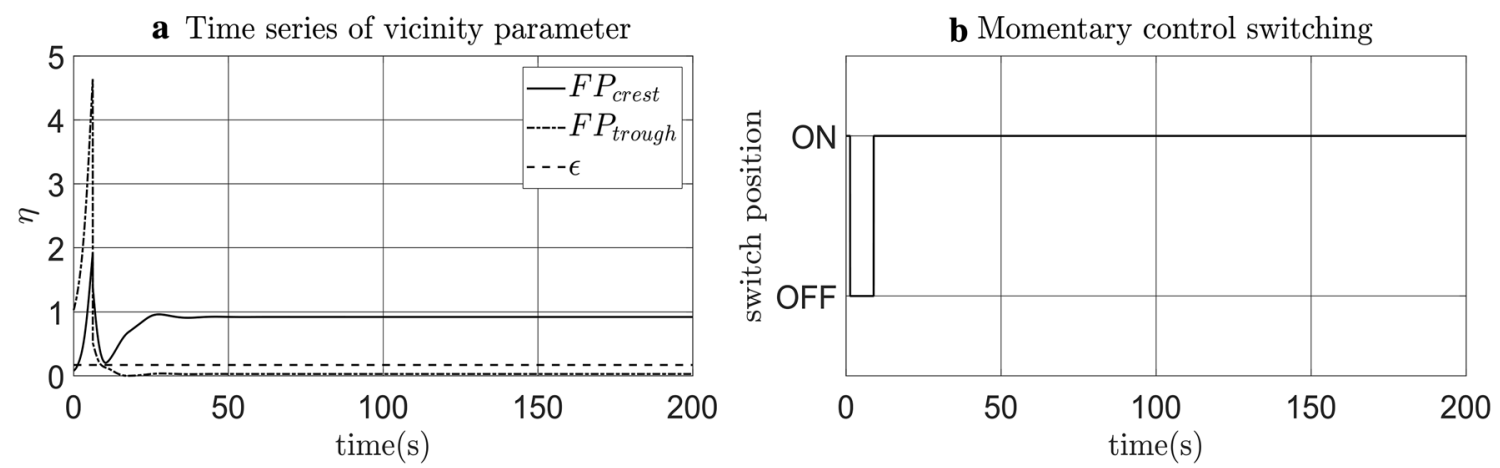

Fig. 8 Case II: Time history of vicinity parameter and control switch

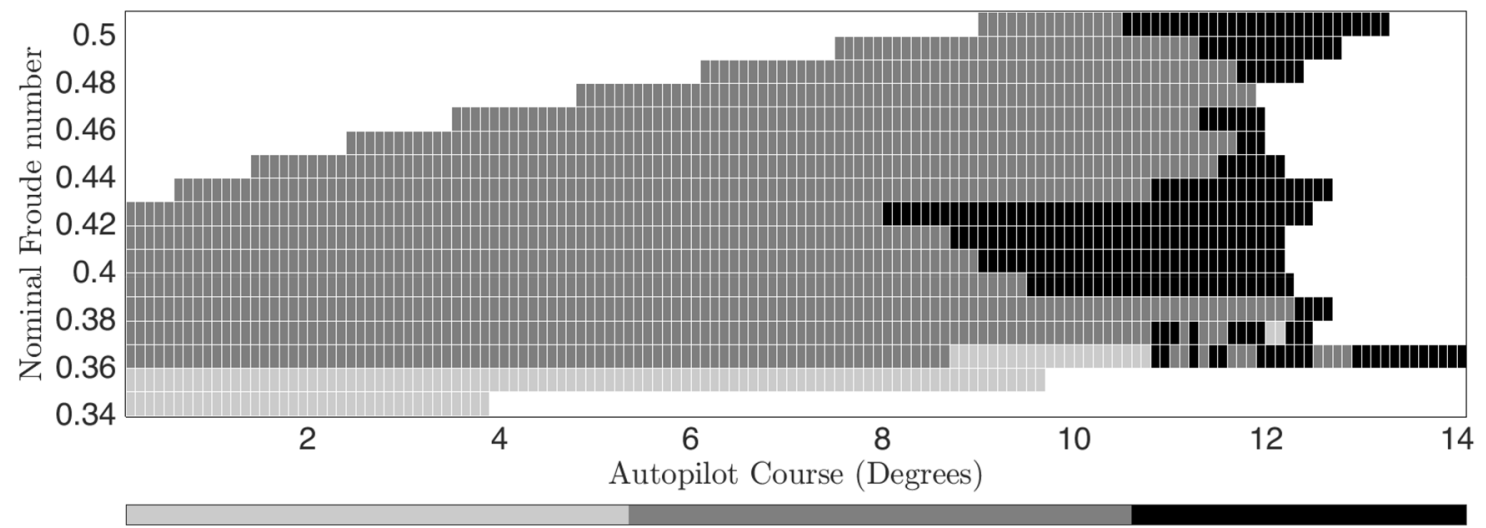

Periodic motion

Surf-riding

Broaching-to and capsize

Fig. 9 Modes of motion observed under the proposed control model with $\lambda / L=1.637$ and $H / \lambda=0.1$ 
broaching-to prevention is primarily achieved by controlling the surge motion happening in the wave downslope, i.e. dropping the propeller thrust near the wave crest fixed point thereby reducing the large yaw moment and enabling the rudder to control the yawing and then raising the same near the wave trough to enter into surf-riding. Hence the influence of rps control is dominant and effective in the success region of autopilot course in Fig. 9. In higher heading angles the large yawing was still uncontrollable with the proposed control model.

\section{Robustness and effectiveness of the controller}

An efficient controller needs to be robust to modeling imprecisions associated with the omission of higher-order dynamics, inaccurate system parameters, and so on. To check the robustness of the controller concerning modeling imprecisions, the authors ignore nonlinear terms of calm-water manoeuvring forces for the momentary state feedback controller i.e., the feedback is evaluated using a less precise model, but the actually simulated system remains as the original model considering these nonlinear

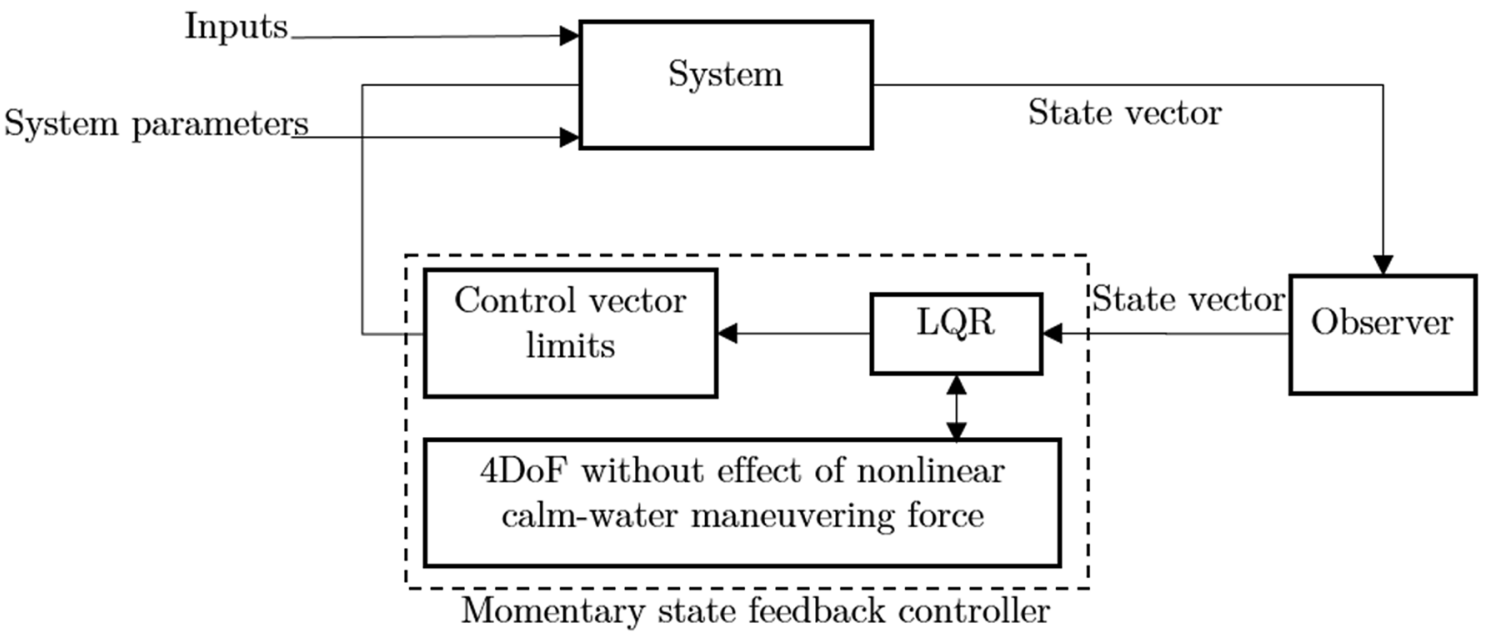

Fig. 10 Schematic of closed-loop system model with modified controller model

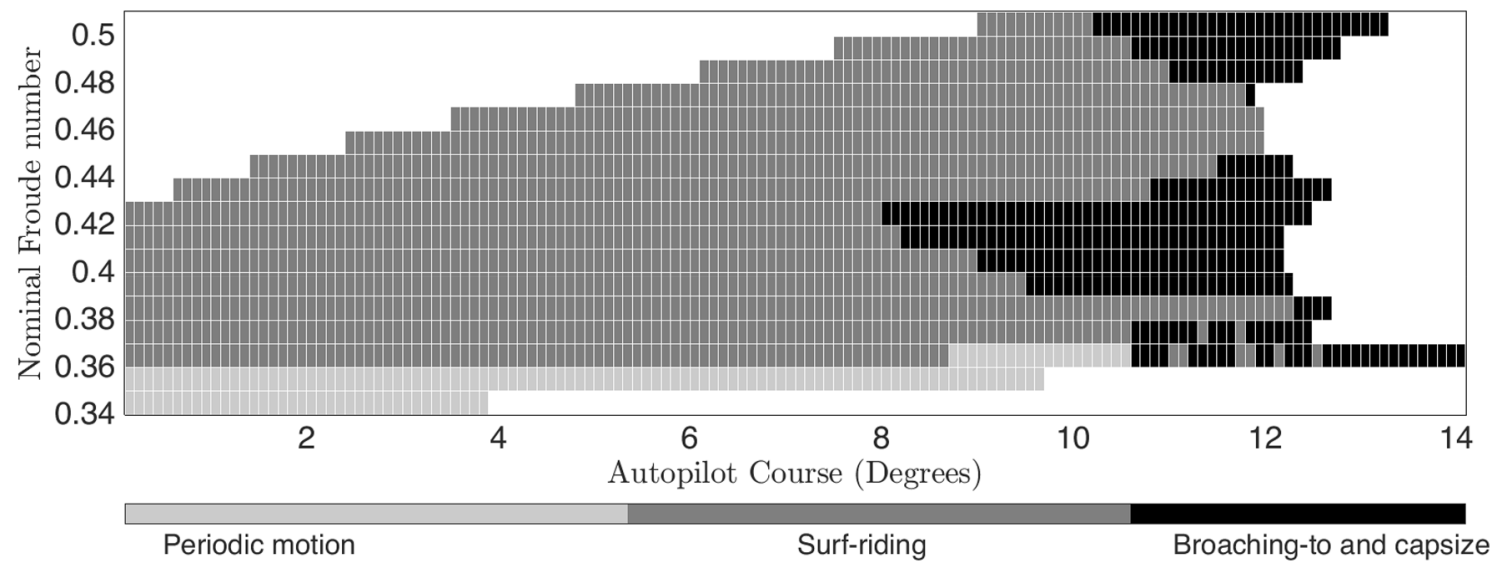

Fig. 11 Modes of motion observed under the modified proposed control model with modeling imprecision and wave condition of $\lambda / L=1.637$ and $H / \lambda=0.1$ 


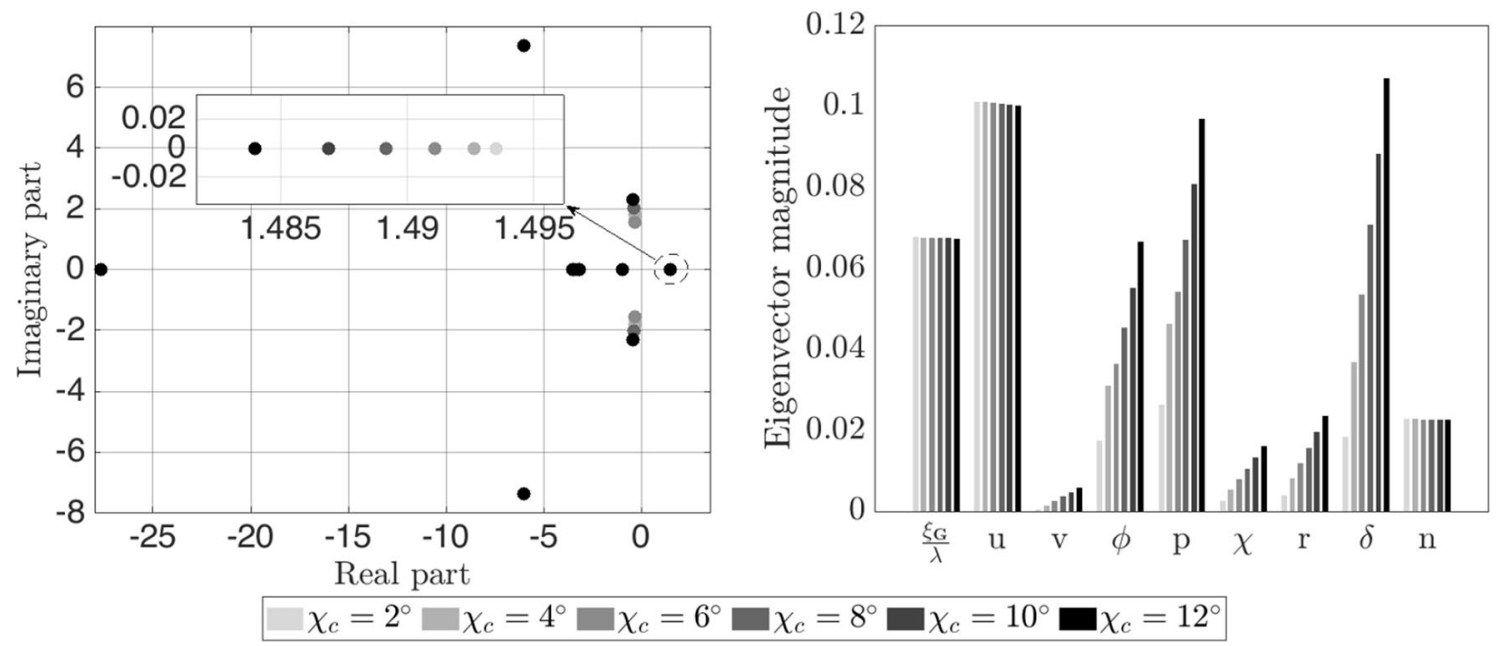

Fig. 12 Eigenvalues and eigenvector trend with the increase of heading angle for a constant Froude number $(F n=0.41)$ at a fixed point near wave crest

terms of calm-water manoeuvring forces. Figure 10 shows the schematic representation of the momentary state feedback controller with the modified controller model. In this simulation model, the fixed points and the momentary state feedback are calculated ignoring the nonlinear manoeuvring force modeling in the new controller model.

Numerical simulation is carried out using the modified controller model for the cases shown in Fig. 9. Figure 11 shows the summary of this simulation.

Figure 11 demonstrates the robustness of the approach with respect to modeling imprecisions. Broaching-to prevention was achieved in lower Froude numbers by undergoing periodic motion and by surf-riding in higher Froude numbers in lower heading angles. As the desired heading angle increases, there is a clear trend that it becomes difficult to prevent broaching-to. Figure 12 shows the trend of eigenvalues and eigenvectors for a high Froude number case with varying autopilot courses. With an increase of heading angle there is only a slight decrease in the magnitude of the positive eigenvalue but the magnitude of the eigenvector corresponding to this positive eigenvalue increases significantly in the roll and yaw directions. The change of heading angle influences more on the swayyaw-roll directions than the surge direction vector field $\left(\dot{\xi}_{G} / \lambda, \dot{u}, \dot{n}\right)$. The failure of the proposed control model in higher heading angles could be attributed to this increased instability in yaw and roll. The controller is mainly able to control the surge instability, but at higher heading angles this was not found to be sufficient for broaching-to prevention. A larger steering force using a high-performance steering gear to counter the yaw moment shall be required in this region.
The effect of the inaccuracy of the system parameter observation on the proposed control model is also investigated. The role of the observer in Fig. 10 is to evaluate the vicinity parameter and to feed the state vector, $\boldsymbol{x}=\left\{\xi_{G} / \lambda, u, v, \phi, p, \chi, r, \delta, n\right\}$ to the controller. Here, $\xi_{G} / \lambda$, the position of the ship center of gravity on the wave is a difficult quantity to be measured in the physical system onboard. To check the performance of the controller without observing the $\xi_{G} / \lambda$, it is omitted from the observer in evaluating the vicinity parameter and from the controller for evaluating the feedback. Hence, the LQR control is active only when the state variables $\{u, v, \phi, p, \chi, r, \delta, n\}$ approaches the surf-riding equilibrium. Only periodic motion cases shown in Fig. 9 are considered here because only in such cases the controller is primarily found effective and $\xi_{G} / \lambda$ will be required in higher Froude number cases to decide on the nature of feedback depending on proximity to a wave crest or trough. However, it should be noted that the $\xi_{G} / \lambda$ is still used in wave force and righting arm calculations. The simulation results with this modified model shows a similar region of periodic motion as shown in Fig. 11. This could be because the surge velocity and $\xi_{G} / \lambda$ approach their fixedpoint values in the same manner and hence measure of one of them could indicate the proximity to saddle point.

The comparison between Figs. 9 and 11 shows the robustness of the controller in dealing with modeling uncertainties, and its effectiveness especially in the lower Froude number region to prevent broaching-to by undergoing periodic motion. This indicates that avoiding broaching at the lower Froude number can be realized even without directly observing the relative position of the ship to waves. 


\section{Conclusions and future works}

The momentary state feedback control focusing on the saddle point in the system is newly proposed to prevent broaching-to in real-time. It was numerically confirmed in regular waves that controlling the surging in a wave downslope during broaching-to enables to change the broaching to a periodic motion at lower Froude numbers. At higher Froude numbers, broaching-to prevention is achieved by undergoing stable surfriding near the wave trough in lower heading angles. However, at a higher Froude number and in a larger heading angle, the instability in yaw direction increases, and the controller was found not to be effective in such cases. Even if the estimated data of nonlinear manoeuvring forces or the observed data of the relative ship position to waves $\left(\xi_{G} / \lambda\right)$ are missing, the momentary state feedback control provides a similar capability to prevent broaching-to, so that this control is robust. The mechanism of broaching-to prevention at high and low Froude number is consistent with the findings of previous work by the authors [1]. However, there is a limitation in the mechanism of broaching-to prevention here at higher Froude numbers due to the engine power especially in longer waves.

An extension of this momentary state feedback control to irregular waves is a future research task. In practice, the knowledge of ship position on waves $\left(\xi_{G} / \lambda\right)$ will require the use of proxy variables such a pitch angle or mean wave celerity, along with the use of wave radar [22] to measure the encountering wave spectrum. The narrow-banded nature of ocean waves and those leading to broaching-to will further aid such an approach. Before moving to simulation in irregular wave, investigating the effectiveness of this approach for more regular wave conditions and extending to irregular waves using a probabilistic approach will be carried out. Furthermore, for preventing dangerous phenomena with larger heading in stern quartering waves with higher speed, it should be investigated together with yaw-roll instability without surfriding and the bow-diving phenomena [23] in the future.

Acknowledgements This work was supported by a Grant-in-Aid for Scientific Research from the Japan Society for Promotion of Science (JSPS KAKENHI Grant Number 19H02360).

Open Access This article is licensed under a Creative Commons Attribution 4.0 International License, which permits use, sharing, adaptation, distribution and reproduction in any medium or format, as long as you give appropriate credit to the original author(s) and the source, provide a link to the Creative Commons licence, and indicate if changes were made. The images or other third party material in this article are included in the article's Creative Commons licence, unless indicated otherwise in a credit line to the material. If material is not included in the article's Creative Commons licence and your intended use is not permitted by statutory regulation or exceeds the permitted use, you will need to obtain permission directly from the copyright holder. To view a copy of this licence, visit http://creativecommons.org/licenses/by/4.0/.

\section{References}

1. Maniyappan S, Umeda N, Maki A, Akimoto Y (2020) Effectiveness and mechanism of broaching-to prevention using global optimal control with evolution strategy (CMA-ES). J Mar Sci Technol. https://doi.org/10.1007/s00773-020-00743-4

2. Ott E, Grebogi C, Yorke JA (1990) Controlling chaos. Phys Rev Lett 64:1196-1199. https://doi.org/10.1103/PhysRevLett.64.1196

3. IMO (1995) Guidance to the master for avoiding dangerous situation in following and quartering seas. MSC/Circ 707

4. IMO (2008) International code on intact stability. MSC 85/26/Add 1 Annex:57

5. Umeda N, Renilson M (1992) Broaching- A dynamic analysis of yaw behaviour of a vessel in a following Sea. In: Wilson PA (ed) Manoeuvering and control of marine craft. Computational Mechanics Publications, Southampton, pp 533-543

6. Spyrou KJ (1995) Surf-riding and oscillations of a ship in quartering waves. J Mar Sci Technol 1:24-36. https://doi.org/10.1007/ BF01240010

7. Renilson M (1986) The seabrake: A device for assisting in the prevention of broaching-to. In: Third International Conference on stability of ships and ocean vehicles. pp 75-80

8. Renilson M (1982) An Investigation into the factors affecting the likelihood of broaching-to in following seas. In: Second International Conference on Stability of Ships and Ocean Vehicles. pp $551-564$

9. Umeda N, Matsuda A, Takagi M (1999) Model experiment on anti-broaching steering system. J Soc Nav Archit Japan 1999:4148. https://doi.org/10.2534/jjasnaoe1968.1999.41

10. Maki A, Umeda N, Ueno S (2008) Investigation on broaching-to using optimal control theory. J Japan Soc Nav Archit Ocean Eng 8:115-122. https://doi.org/10.2534/jjasnaoe.8.115

11. Hansen N (2016) The CMA evolution strategy: a tutorial

12. Maki A, Sakamoto N, Akimoto $Y$ et al (2021) On broaching-to prevention using optimal control theory with evolution strategy (CMA-ES). J Mar Sci Technol 26:71-87. https://doi.org/10.1007/ s00773-020-00722-9

13. Kirk DE (1988) Section 5.3 Linear regulator problems. In: Optimal control theory. pp 209-227

14. ITTC (2002) The specialist committee on prediction of extreme ship motions and capsizing. Proc 23rd Int Towing Tank Conf Volume II:619-748

15. Makino H, Umeda N, Ohtsuka T et al (2017) Energy savings for ship propulsion in waves based on real-time optimal control of propeller pitch and electric propulsion. J Mar Sci Technol 22:546558. https://doi.org/10.1007/s00773-017-0434-1

16. Umeda N, Hashimoto H (2002) Qualitative aspects of nonlinear ship motions in following and quartering seas with high forward velocity. J Mar Sci Technol 6:111-121. https://doi.org/10.1007/ s007730200000

17. Umeda $N$ (1999) Nonlinear dynamics of ship capsizing due to broaching in following and quartering seas. J Mar Sci Technol 4:16-26. https://doi.org/10.1007/s007730050003

18. Strogatz HS (1994) Hyperbolic fixed points, topological equivalence and structural stability. In: Nonlinear dynamics and chaos. Perseus Books, p 155

19. Ohtsu K, Hasegawa K (1981) Evaluation and perspectives of autopilot. In: Third symposium on ship maneuverability. The Society of Naval Architects of Japan, Tokyo, pp 243-279

20. Anderson BDO, Moorer JB (1989) Section 5.3 -5.5 Some classical control ideas: sesitivity, complementary sensitivity, and Robustness. In: Optimal control. Prentice-Hall International, Inc., pp $110-131$

21. Umeda N (2000) Application of nonlinear dynamical system approach to ship capsize due to broaching in following and 
quartering seas. In: Contemporary ideas on ship stability. Elsevier Science Publications (Amsterdam), pp 57-68

22. Yano T, Umeda N, Hirayama K, et al (2019) Wave radar application to the simplified parametric roll operational guidance at actual sea. In: 17th International ship stability workshop. pp 91-96

23. Umeda N, Hamamoto M (2000) Capsize of ship models in following/quartering waves: physical experiments and nonlinear dynamics. Philos Trans R Soc London Ser A Math Phys Eng Sci 358:1883-1904. https://doi.org/10.1098/rsta.2000.0619

Publisher's Note Springer Nature remains neutral with regard to jurisdictional claims in published maps and institutional affiliations. 